\title{
Non-invasive Intravascular Pressure Gradient Estimation using Synthetic Aperture Ultrasound
}

Haslund, Lars Emil; Kurukuladithya, Shamal Surain ; Ariyasinghe, Malmindi ; Stuart, Matthias Bo; Traberg, Marie Sand; Jensen, Jørgen Arendt

\section{Published in:}

Proceedings of 2021 IEEE International Ultrasonics Symposium

Link to article, DOI:

10.1109/IUS52206.2021.9593766

Publication date:

2021

Document Version

Peer reviewed version

Link back to DTU Orbit

Citation (APA):

Haslund, L. E., Kurukuladithya , S. S., Ariyasinghe, M., Stuart, M. B., Traberg, M. S., \& Jensen, J. A. (2021). Non-invasive Intravascular Pressure Gradient Estimation using Synthetic Aperture Ültrasound. In Proceedings of 2021 IEEE International Ultrasonics Symposium IEEE. https://doi.org/10.1109/IUS52206.2021.9593766

\section{General rights}

Copyright and moral rights for the publications made accessible in the public portal are retained by the authors and/or other copyright owners and it is a condition of accessing publications that users recognise and abide by the legal requirements associated with these rights.

- Users may download and print one copy of any publication from the public portal for the purpose of private study or research.

- You may not further distribute the material or use it for any profit-making activity or commercial gain

- You may freely distribute the URL identifying the publication in the public portal 


\title{
Non-invasive Intravascular Pressure Gradient Estimation using Synthetic Aperture Ultrasound
}

\author{
Lars Emil Haslund ${ }^{1}$, Shamal Surain Kurukuladithya ${ }^{1}$, Malmindi Ariyasinghe ${ }^{1}$, Matthias Bo Stuart ${ }^{1}$, \\ Marie Sand Traberg ${ }^{1}$, Jørgen Arendt Jensen ${ }^{1}$, IEEE Fellow \\ ${ }^{1}$ Center for Fast Ultrasound Imaging, Department of Health Technology, \\ Technical University of Denmark, DK-2800 Lyngby, Denmark
}

\begin{abstract}
This study presents a new method for noninvasive pressure gradient estimations. It combines a new method for estimating the spatio-temporal acceleration of the flowing blood with the Navier-Stokes equation. The method is based on a double cross-correlation approach, which is hypothesized to generate more precise pressure gradients than previous methods. The acceleration is determined by cross-correlating the correlation functions attained from the velocity estimation. Data are acquired using a $5.2 \mathrm{MHz}$ linear transducer in combination with a Verasonics research scanner. A permuted, pulse inverted, interleaved sequence with four virtual sources evenly distributed on the aperture is used. The performance of the method is tested on experimental data, measured on a bifurcation phantom of the common carotid artery. The pressure drop across nine flow cycles varied from -20 Pa to $40 \mathrm{~Pa}$, with a standard deviation of $15.7 \%$.
\end{abstract}

\section{INTRODUCTION}

Intravascular pressure gradient estimation is an important metric for monitoring the cardiovascular system and the development of cardiovascular diseases such as atherosclerosis [1]. The measurement is often acquired by an intravascular pressure sensitive catheter. The procedure is cumbersome, invasive and not without risk for the patients. De Vecchi et al. [2] showed that invasive catheters overestimates the peak systolic pressure with up to $24 \%$ compared to the gold standard using a wire. They found that the presence of the catheter was the primary cause for the overestimation. In 1976, Holen et al. [3] was the first to introduce a non-invasive ultrasound based method for estimating pressure gradients in mitral stenosis. Since then, more advanced methods have been developed in ultrasound [4] and within MRI [5] [6].

\section{THEORY}

In this study pressure gradients are calculated from Navier-Stokes equation:

$$
\rho\left[\frac{\partial \vec{v}}{\partial t}+\vec{v} \cdot \nabla \vec{v}\right]=-\nabla p+\rho \vec{g}+\mu \nabla^{2} \vec{v}
$$

It relates the forces acting on an incompressible fluid volume to its acceleration and density. In (1) the left hand side describes the acceleration, where $\rho$ is the fluid density, $\vec{v}$ is the velocity field and $\nabla$ is the del operator. The right hand side describes the forces acting on the fluid, where $\nabla p$ is the change in pressure, $\vec{g}$ is the gravitational force, and $\mu$ is the fluid viscosity. NavierStokes equation can be further simplified by omitting the influence of both viscosity and gravity. The viscous term has previously been shown to have little influence in large blood vessels [4] [7]. Gravity can be neglected as the phantom undergoing examination is placed in a supine position. Finally, rewriting the equation to a scalar equation following a streamline gives [4]:

$$
\frac{\partial p}{\partial s}=-\rho\left[\frac{\partial v_{s}}{\partial t}+v_{s} \frac{\partial v_{s}}{\partial s}\right] \text {. }
$$

Here, $v_{s}$ is a scalar product between $\vec{v}(\vec{r}, t)$ and the unit vector that lies tangential to the streamline $\vec{s}$, and $d \vec{s}$ denotes an element distance along the streamline that runs in the direction of $\vec{s}$. Finally, integrating (2) along a streamline yields the pressure difference:

$$
\Delta p(t)=\int_{0}^{L} \frac{\partial p}{\partial s} d s
$$

\section{METHOD}

The pressure differences are dependent on both temporal and spatial acceleration, which can be derived from the velocity field. Reliable calculation of accelerations directly influences the precision and accuracy of the pressure gradient estimates. This section describes a double cross-correlation method for accurate calculation of both the temporal and spatial acceleration. The sequence and velocity estimation is first described, and then the acceleration estimation is given.

\section{A. Sequence}

Synthetic aperture (SA) ultrasound imaging is performed by using a permuted, pulse inverted, interleaved 
sequence with four virtual sources evenly distributed on the aperture [8]. This sequence uses eight emissions for making two high-resolution images, which are $T_{p r f}$ apart. The sequence enables $T_{p r f}$ temporal resolution, which is desired when performing velocity estimations for high velocities. Table I shows how each virtual source emits two pulses after each other.

\section{TABLE I: Sequence}

\begin{tabular}{|l|l|l|l|l|l|l|l|l|}
\hline Virtual source & \multicolumn{2}{|c|}{1} & \multicolumn{2}{|c|}{2} & \multicolumn{2}{|c|}{3} & \multicolumn{2}{|c|}{4} \\
\hline Positive emissions & 1 & & 3 & & 5 & & 7 & \\
\hline Negative emissions & & 2 & & 4 & & 6 & & 8 \\
\hline
\end{tabular}

\section{B. Stationary Echo Cancellation}

The scattering coefficients of vessel boundaries are often 10 to 100 times larger than for blood cells [9]. Hence, the signal contribution emanating from the stationary tissue must be removed prior to blood velocity estimations. The stationary signal $y_{\text {sta }}(j)$ can be calculated by taking the mean of $N$ rf-signals:

$$
y_{s t a}(j)=\frac{1}{N} \sum_{i=0}^{N-1} y(j, i) .
$$

Here, $y(j, i)$ denotes a beamformed signal, where each signal is a function of space $j$ and emission number $i$ (time). The stationary component is then removed by subtracting it from each signal as:

$$
y_{e}(j, i)=y(j, i)-y_{s t a}(j) .
$$

Here, $y_{e}(j, i)$ denotes the echo-canceled signal.

\section{Velocity Estimation}

The blood velocity is found using the directional cross-correlation method [10]. The discrete crosscorrelation $R_{12}$ of two temporally spaced signals $y_{e}(j, i)$ and $y_{e}(j, i+1)$ can be described by the following equation:

$$
\begin{aligned}
R_{12}(k) & =\sum_{j=0}^{L-1} y_{e}(j, i) y_{e}(j+k, i+1), \\
k & =0, \pm 1, \pm 2, \pm 3 \ldots \pm L-1
\end{aligned}
$$

where $L$ is the sample length of the analyzed line segment and $k$ denotes the lag. The displacement of the scatterers is then estimated from the shift in peak position of the cross correlation function. Since the shift in peak position is often lower than the lag resolution, polynomial interpolation is needed. This is performed by fitting a second order polynomial to the three points at the peak of the cross correlation. The interpolated peak position can then be determine from the polynomial [9]:

$$
k_{s}=k_{p}-\frac{R_{12}\left(k_{p}+1\right)-R_{12}\left(k_{p}-1\right)}{2\left(R_{12}\left(k_{p}+1\right)-2\left(R_{12}\left(k_{p}\right)\right)+R_{12}\left(k_{p}-1\right)\right.},
$$

where, $k_{p}$ denotes the peak position from the cross correlation $R_{12}$ and $k_{s}$ describes the position of the interpolated peak. The blood velocity is then given by:

$$
v_{\theta}=\frac{k_{s} \cdot \Delta x}{T_{p r f} \cdot N_{t}}
$$

where $\Delta x$ is the spatial sampling interval in units of meters, $T_{p r f}$ is the time between pulse repetitions, and $N_{t}$ is the number of emissions between the two signals correlated.

\section{Acceleration Estimation}

Fig. 1 shows a simple schematic describing the principle behind the temporal acceleration estimator. Here, cross-correlation functions from velocity estimates acquired at different times, at the same position, are crosscorrelated again. The lag shift of the resulting crosscorrelation function is proportional to the temporal acceleration. The spatial acceleration estimator computes

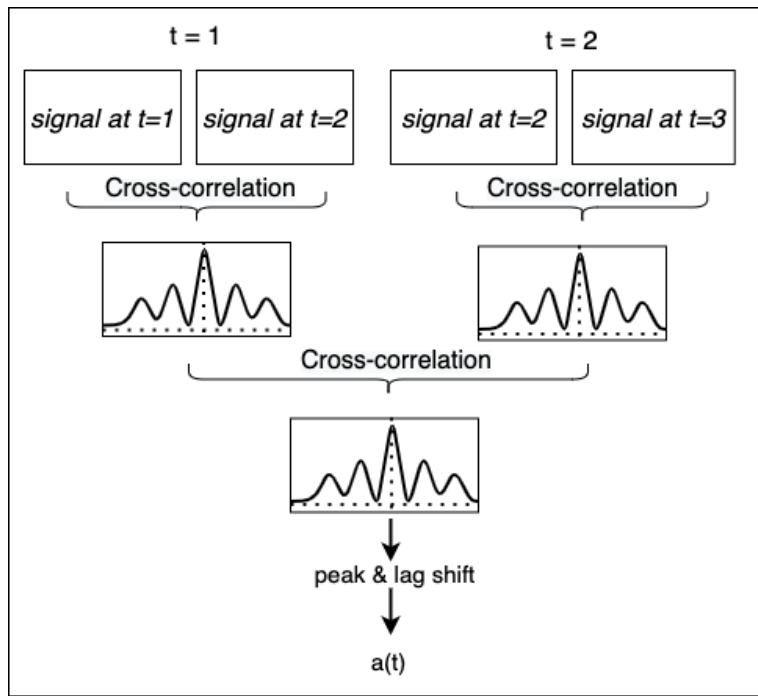

Fig. 1: Shows the principle behind the double crosscorrelation method for the temporal acceleration.

cross-correlation functions from velocity estimates acquired at the same time, but at different positions. The temporal and spatial acceleration for the used sequence are:

$$
\begin{aligned}
A c c_{t} & =\frac{k_{s} \Delta x}{N_{e} K_{t}\left(T_{p r f} \cdot N_{t}\right)^{2}} . \\
A c c_{s} & =\frac{k_{s} \Delta x}{K_{s} \Delta r\left(T_{p r f} \cdot N_{t}\right)} .
\end{aligned}
$$


Here, $k_{s}$ is the lag shift obtained by the double crosscorrelation approach, $\Delta x$ is the spatial sampling interval, and $K_{t}$ and $K_{s}$ denotes the temporal and spatial distance between correlation functions [11]. $N_{t}$ is the number of emissions separating two correlation signals, and $N_{e}$ is the number of emissions separating correlation pairs. At last $\Delta r$ denotes the spatial distance separating the two adjacent points, for the correlation functions.

\section{EXPERIMENTAL SETUP}

A bifurcation phantom, based on magnetic resonance imaging data from a healthy volunteer, is used. The phantom was connected to a pulsating flow pump (CompuFlow 100, shelley Medical Imaging Technologies, Toronto, Canada). The volume profile used during measurement was set to mimic flow in the carotid artery with a peak flow rate of $12.9 \mathrm{ml} / \mathrm{s}$.

\section{A. Data Acquisition}

A 5.2 MHz linear transducer (BK medical Aps, Herlev, Denmark) was positioned on top of the phantom. Data are obtained with a Vantage research scanner (Verasonics, Kirkland, Washington, USA) with a pulse repetition frequency of $5 \mathrm{kHz}$. The frame rate for the correlation pairs was initially $625 \mathrm{~Hz}$. However, by applying recursive SA imaging this was increased to $2500 \mathrm{~Hz}$ [8]. Table II contains the acquisition parameters for the sequence.

TABLE II: Acquisition parameters.

\begin{tabular}{l|c|c|c} 
Parameter & Value & Unit & Variable \\
\hline No. elements & 192 & - & - \\
\hline Centre frequency & 5.2 & $\mathrm{MHz}$ & - \\
\hline Element pitch & 0.2 & $\mathrm{~mm}$ & - \\
\hline Pulse repetition frequency & 5 & $\mathrm{kHz}$ & $f_{\text {prf }}$ \\
\hline Frame rate(recursive) & 2500 & $\mathrm{fr} / \mathrm{s}$ & - \\
\hline Transmit F-number & -0.7 & - & - \\
\hline Vessel diameter & 6 & $\mathrm{~mm}$ & - \\
\hline Measurement depth & 33.5 & $\mathrm{~mm}$ & - \\
\hline Spatial sampling interval & 0.0185 & $\mathrm{~mm}$ & $\Delta x$
\end{tabular}

\section{B. Data Processing}

Six points separated by $2 \mathrm{~mm}$ are placed at a depth of $33.5 \mathrm{~mm}$ in the center of the common carotid artery, see Fig. 2. They are the center point for the directional rf-lines. The length of the directional lines is $6 \mathrm{~mm}$. The directional lines are then positioned in the flow direction. Prior to the cross-correlation procedure, a Hann window is applied to the directional rf-signals. This weights the central part of the line segments. When calculating the velocity, temporal averaging is performed across $40 \mathrm{~ms}$.
For the acceleration estimates the temporal and spatial distances are set to $K_{t}=40$ and $K_{s}=1$. Finally, averaging in depth is performed to increase the precision of the velocity estimates. Table III contains the data processing parameters.

TABLE III: Data processing parameters

\begin{tabular}{l|c|c|c} 
Parameter & Value & Unit & Variable \\
\hline Distance between points & 2 & $\mathrm{~mm}$ & $\Delta r$ \\
\hline Measuring depth & 33.5 & $\mathrm{~mm}$ & - \\
\hline Line length & 6 & $\mathrm{~mm}$ & - \\
\hline Echo canceling & 8 & $\mathrm{fr}$ & $N$ \\
\hline Weighting function & Hann window & - & \\
\hline Temporal averaging & 100 & $\mathrm{~ms}$ & - \\
\hline Temporal skipping step & 40 & - & $K_{t}$ \\
\hline Spatial skipping step & 1 & - & $K_{s}$ \\
\hline $\begin{array}{l}\text { Emissions separating two } \\
\text { correlation frames }\end{array}$ & 1 & $\mathrm{em}$ & $N_{t}$ \\
\hline $\begin{array}{l}\text { Emissions separating cor- } \\
\text { relation pairs (recursive) }\end{array}$ & 2 & $\mathrm{em}$ & $N_{e}$ \\
\hline Wavelength & 0.2962 & $\mathrm{~mm}$ & $\lambda$ \\
\hline Spatial averaging in depth & {$[-\lambda / 2: \lambda / 4: \lambda / 2]$} & $\mathrm{mm}$ & - \\
\hline
\end{tabular}

\section{REsults AND Discussion}

Fig. 2 shows a B-mode image of the blood vessel. Six points along a streamline are selected and used as an origin for the directional rf-lines. The velocity and temporal acceleration are then calculated at each point and the spatial acceleration are calculated between two adjacent points. This is then used in (2) and (3) to get the pressure difference along the streamline. Fig. 3 shows the pressure drop as a function of time across nine aligned flow cycles. It is seen that the pressure difference along the streaming varies from $-20 \mathrm{~Pa}$ to $40 \mathrm{~Pa}$ with a standard deviation of $15.7 \%$. These result are based on a 1-D velocity estimator. Thus, both the out-of-plane motion, and the axial motion have been excluded. Depending on the complexity of the flow, this will influence the precision of the results. As previously stated the viscosity is neglected, because it has little influence in the center of a large blood vessel. However, the viscosity should be included, when examining smaller blood vessels. Finally, temporal averaging should ideally be performed over the shortest time interval as possible, as it may average out information related to the pressure changes. This study performed a running temporal average across $40 \mathrm{~ms}$ for the velocity estimates.

\section{CONCLUSION}

This paper has introduced a new method for calculating the pressure difference along a streamline in a large phantom blood vessel. It relies on a double 


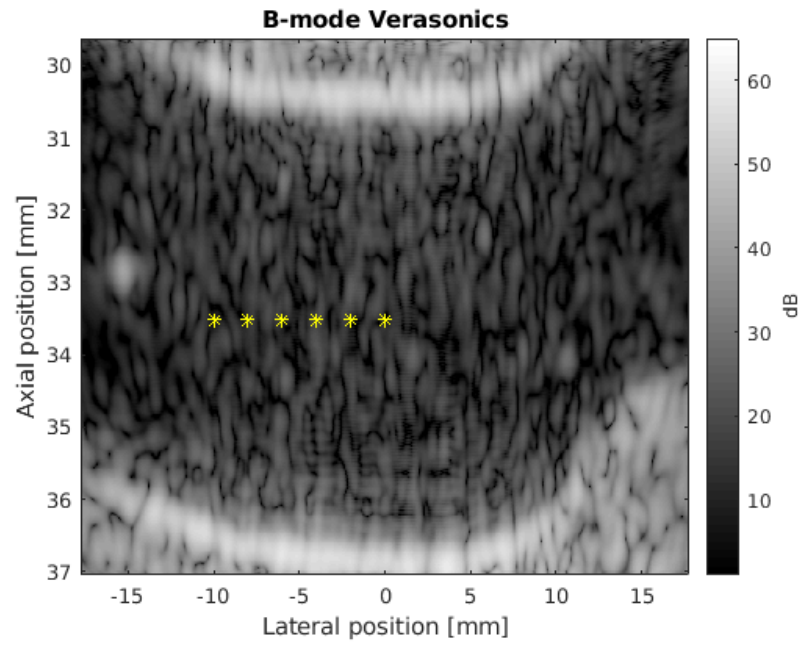

Fig. 2: This figure shows a B-mode image of a phantom blood vessel with the location of six points used for pressure gradient estimations. The points are located proximal to the bifurcation, which is located on the left side of the image.

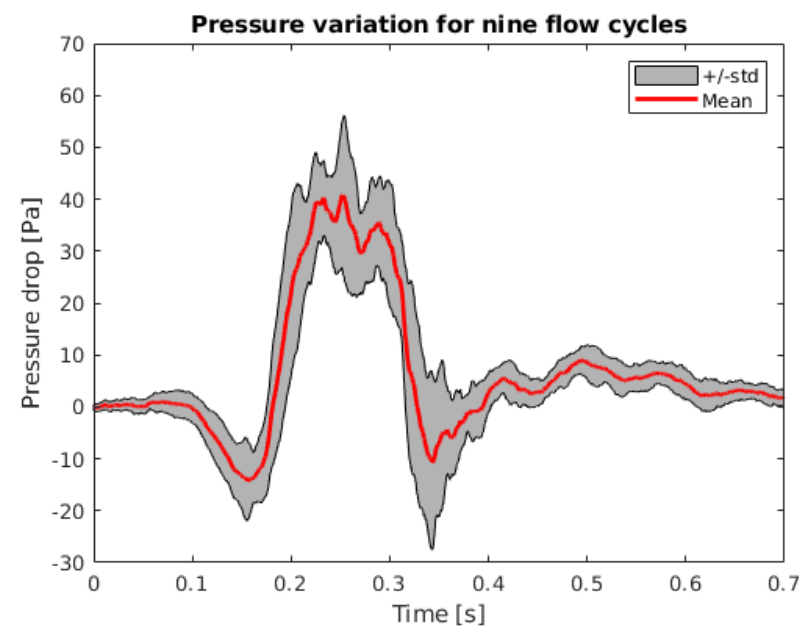

Fig. 3: Shows the pressure variation across nine heart cycles. It is seen that the pressure variation goes from -20 $\mathrm{Pa}$ to $40 \mathrm{~Pa}$. The red line shows the mean pressure variation and the shaded area defines the standard deviation.

cross correlation approach, which allows acceleration estimates to be calculated directly from beamformed ultrasound data. The precision of the method was found to be $15.7 \%$ across nine flow cycles. Future work should investigate a method for evaluating the accuracy. Here, pressure sensitive catheters could be used as a reference model along with finite element models.

\section{REFERENCES}

[1] D. S. Baim and W. Grossman, Grossman's cardiac catheterization, angiography, and intervention. Lippincott Williams \& Wilkins, 2000.

[2] A. de Vecchi, R. E. Clough, N. R. Gaddum, M. C. M. Rutten, P. Lamata, T. Schaeffter, D. A. Nordsletten, and N. P. Smith, "Catheter-induced errors in pressure measurements in vessels: An in-vitro and numerical study," IEEE Trans. Biomed. Eng., vol. 61, no. 6, pp. 1844-1850, 2014.

[3] J. Holen, R. Aaslid, and K. Landmark, "Determination of pressure gradient in mitral stenosis with a non-invasive ultrasound Doppler technique," Acta med. scand., vol. 32, pp. 455-460, 1976.

[4] J. B. Olesen, C. A. Villagómez Hoyos, N. D. Møller, C. Ewertsen, K. L. Hansen, M. B. Nielsen, B. Bech, L. Lönn, M. S. Traberg, and J. A. Jensen, "Non-invasive estimation of pressure changes using 2-D vector velocity ultrasound: An experimental study with in-vivo examples," IEEE Trans. Ultrason., Ferroelec., Freq. Contr., vol. 65, no. 5, pp. 709-719, 2018.

[5] J. Zhang, M. C. Brindise, S. Rothenberger, S. Schnell, M. Markl, D. Saloner, and V. L. Rayz, "4d flow mri pressure estimation using velocity measurement-error-based weighted least squares," IEEE Trans. Med. Imag., vol. 39, no. 5, pp. 1668-1680, 2020.

[6] T.-Q. Nguyen, K. L. Hansen, T. Bechsgaard, L. L. Ann, J. A. Jensen, and M. B. Nielsen, "Non-invasive assessment of intravascular pressure gradients: A review of current and proposed novel methods," diagnostics, 2018.

[7] N. B. Wood, "Aspects of fluid dynamics applied to the large arteries," J. Theor. Biol., vol. 199, no. 953, pp. 137-161, 1999.

[8] J. A. Jensen, "Estimation of high velocities in synthetic aperture imaging: I: Theory," IEEE Trans. Ultrason., Ferroelec., Freq. Contr., vol. 66, no. 6, pp. 1024-1031, 2019.

[9] —-, Estimation of Blood Velocities Using Ultrasound: A Signal Processing Approach. New York: Cambridge University Press, 1996.

[10] J. Kortbek and J. A. Jensen, "Estimation of velocity vector angles using the directional cross-correlation method," IEEE Trans. Ultrason., Ferroelec., Freq. Contr., vol. 53, pp. 20362049, 2006.

[11] J. B. Olesen, J. A. Jensen, and C. A. Villagomez-Hoyos, "Flow acceleration estimation directly from beamformed ultrasound data," 2018, patent Application PCT/IB2015/055592. 